\title{
Co-activation of upper limb muscles during reaching in post-stroke subjects: An analysis of the contralesional and ipsilesional limbs
}

\author{
Cláudia C. Silva ${ }^{\mathrm{a}, *}$, Augusta Silva $^{\mathrm{a}}$, Andreia Sousa ${ }^{\mathrm{a}}$, Ana Rita Pinheiro ${ }^{\mathrm{a}}$, Catarina Bourlinova ${ }^{\mathrm{d}}$, Ana Silva $^{\mathrm{b}}$, \\ António Salazar ${ }^{\mathrm{b}}$, Carla Borges ${ }^{\mathrm{b}}$, Carlos Crasto ${ }^{\mathrm{a}}$, Miguel Velhote Correia ${ }^{\mathrm{b}}$, João Paulo Vilas-Boas ${ }^{\mathrm{c}}$, \\ Rubim Santos ${ }^{\text {a }}$
}

\footnotetext{
${ }^{a}$ Centro de Estudos do Movimento e Actividade Humana (CEMAH), ESTSP-IPP, V. N. Gaia, Portugal

b INESC-TEC and Faculty of Engineering, University of Porto, Porto, Portugal

${ }^{\mathrm{c}}$ CIFI2D, Faculty of Sport, and Porto Biomechanics Laboratory, University of Porto, Porto, Portugal

${ }^{\mathrm{d}}$ Escola de Educação Física e Esporte, Universidade de São Paulo, São Paulo, SP, Brazil

* Corresponding author. Address: Área Cientifica da Fisioterapia, Escola Superior de Tecnologia da Saúde do Porto, Instituto Politécnico do Porto, Centro de Estudos do Movimento e Actividade Humana - CEMAH, Faculdade de Desporto da Universidade do Porto, Rua Valente Perfeito, 322, 4400-330 Vila Nova de Gaia, Portugal. Tel.: +351965129399.

E-mail address: ccs@estsp.ipp.pt (C.C. Silva).
}

\begin{abstract}
The purpose of this study was to analyze the change in antagonist co-activation ratio of upper-limb muscle pairs, during the reaching movement, of both ipsilesional and contralesional limbs of post-stroke subjects. Nine healthy and nine post-stroke subjects were instructed to reach and grasp a target, placed in the sagittal and scapular planes of movement. Surface EMG was recorded from postural control and movement related muscles. Reaching movement was divided in two sub-phases, according to proximal postural control versus movement control demands, during which antagonist co-activation ratios were calculated for the muscle pairs LD/PM, PD/AD, TRIlat/BB and TRIlat/BR. Post-stroke's ipsilesional limb presented lower co-activation in muscles with an important role in postural control (LD/PM), comparing to the healthy subjects during the first sub-phase, when the movement was performed in the sagittal plane $(p<0.05)$. Conversely, the post-stroke's contralesional limb showed in general an increased co-activation ratio in muscles related to movement control, comparing to the healthy subjects. Our findings demonstrate that, in post-stroke subjects, the reaching movement performed with the ipsilesional upper limb seems to show co-activation impairments in muscle pairs associated to postural control, whereas the contralesional upper limb seems to have signs of impairment of muscle pairs related to movement.
\end{abstract}

Keywords: Stroke; Reaching; Antagonist co-activation ratio; Ipsilesional limb

\section{Introduction}

Disorders of the central nervous system often result in conditioned motor function as a consequence of atypical patterns of muscle recruitment. Besides being the leading cause of persistent neurological impairment, stroke causes seriously functional limitations, being considered a major health problem with enormous economic implications in several countries all over the world (WHO, 2011).

Currently, there are several accepted rehabilitation approaches based on different principles (Tate, 2006). However, we believe that it is imperative that physiotherapists perform a movement analysis-based clinical reasoning for designing a subject-directed rehabilitation intervention. Therefore, understanding the mechanisms related to atypical versus typical movement pattern is an emerging need, so that decisions regarding the most appropriate rehabilitation strategies can be taken. Moreover, in order to support the clinical reasoning, motor performance assessment should rely on standard and easy applicable instruments. However, standardized clinical motor performance assessment scales are not sensitive enough for the measurement of certain quantitative features (e.g., intersegment coordination, quality and smoothness of movement), not providing accurate information about motor synergies (Patel et al., 2010).

Despite the difficulty of incorporating reliable assessment instruments amongst clinical setting scenarios, surface electromyography (sEMG) seems to represent a valid and relatively easy-to-use tool for motor performance assessment. In fact, signal acquisition through sEMG allows an extensive and in-depth characterization of motor control patterns, which is extremely important to orientate the clinical reasoning process (Hughes et al., 2009, 2010). 
In stroke patients, the contralesional motor deficits have been extensively described (Bobath, 1990; Bourbonnais et al., 1989; Burke, 1988). Nonetheless, based on the current neuroscience knowledge regarding the neurophysiology of movement organization, ipsilesional deficits should also exist, although these have been less described among the clinical and research community (Desrosiers et al., 1996; Sunderland et al., 1999). Indeed, in an unilateral stroke, with the brain lesion located at a sub-cortical level (such as the frequently affected internal capsule), it is highly probable that the lesion interferes with the neuronal connection between the motor cortex and the reticular formation, which clearly justifies an ipsilesional postural control dysfunction (Schepens, 2004; Schepens et al., 2008). Considering the ventromedial system disposal, which, although bilateral, presents a predominant ipsilateral projection, the ipsilesional deficits can no longer be ignored. Thus, research about the motor behavior of the ipsilesional upper limb, should not only address the hand's dexterity, but proximal components as well (i.e. trunk, shoulder and elbow), which constitutes a still unexplored field.

Given that the major role of the ventromedial system is associated with postural control, it is expected to find signs of dysfunction in stability-related muscles. The inherent specificity of postural control study imposes some demands about the selection of the task and the respective sub-phases, as well as the muscles to be studied. For example, to study the reaching movement it becomes important to assess latissimus dorsi and pectoralis major, as opposed to those mainly related to movement execution, such as deltoid or triceps (Dickstein et al., 2004; Geuze, 2005; Zattara et al., 1988). The importance of the evaluation of postural control muscles during movement tasks is sustained by the evidence that purposeful and orientated distal movement requires the ability to recruit proximal stability (Champiom et al., 2009; Shumway-Cook et al., 2007; Yanga et al., 2002). In this sense, the reach movement can be divided in two sub-phases: (a) the first sub-phase, here named as the elbow flexion phase, which includes the period from the movement' beginning until the maximum elbow flexion; this phase requires a highly demanding proximal postural control; and (b) the second sub-phase, here named as the shoulder flexion phase, starts when the elbow reaches its maximum flexion and ends when the target is reached; this phase is predominantly movement demanding (Fig. 1).

As previously stated, the postural control dysfunction occurring at the ipsilesional side of stroke subjects can only be considered when lesions interfere with the cortico-reticular network. At the neuromuscular level, the deregulation of neurophysiologic mechanisms, such as the reciprocal innervation, is one of the alterations that can exist, being expressed with altered co-activation levels between muscle pairs - (antagonist co-activation ratio). Indeed, the mechanism of reciprocal innervation has been highlighted as one of the altered mechanisms following stroke, mainly in the form of high levels of antagonist co-activation ratio (Hammond et al., 1988; Stoeckmann et al., 2009). Despite the large amount of research about co-activation ratios in stroke subjects (Fellows et al., 1994; Higginson et al., 2006; Lamontagne et al., 2002, 2000; Stoeckmann et al., 2009), the study of the behavior of the muscle pairs related to postural versus movement functions has not been explored so far. The researches in this area are mainly focused on an analytical study of shoulder and/or elbow muscle pairs activity, often demonstrating higher levels of co-activation ratios in the contralesional side. However, these studies did not considered the different motor control dimensions (postural control and movement), and because of that, only movement related muscles have been studied (el-Abd et al., 1993; Gowland et al., 1992; Stoeckmann et al., 2009) (Fellows et al., 1994). To the best of our knowledge, no study has assessed the functional behavior of postural control and movement control related muscles in subjects with postural control and movement control dysfunction, such as in the case of post-stroke subjects with lesions at sub-cortical level at the middle cerebral artery (Matsuyama et al., 2004; Silva et al., 2012a,b; Sousa et al., 2013).

Therefore, the purpose of this study was to analyze the change in antagonist co-activation ratio of upper-limb muscles pairs of either ipsilesional and contralesional limbs of stroke subjects during the reaching movement.

\section{Methods}

\subsection{Participants}

Eighteen subjects, included in two distinct groups (healthy, $n=9$, mean age: $52.3 \pm 4.9$, mean body mass index: $26.1 \pm 1.8$; and post-stroke, $n=9$, mean age: $55.0 \pm 9.6$, mean body mass index: $27.1 \pm 4.0$ ), participated in this study. The protocol was approved by the Ethics Committee of Escola Superior de Tecnologia da Saúde do Porto (ESTSP). The investigation conforms to the principles outlined in the Declaration of Helsinki.

For general group's inclusion, subjects must be above 45 years old. Exclusion criteria comprised musculoskeletal pathology, neck and/or upper limb pain, cerebellar, basal ganglia or brain stem lesions, and a Mini Mental State Examination score below 25.

For the post stroke group inclusion, participants must meet the additional following criteria: unilateral stroke at the subcortical

PHASE 1 Maximum Elbow Flexion

PHASE 2 Elbow Extension (reach target)

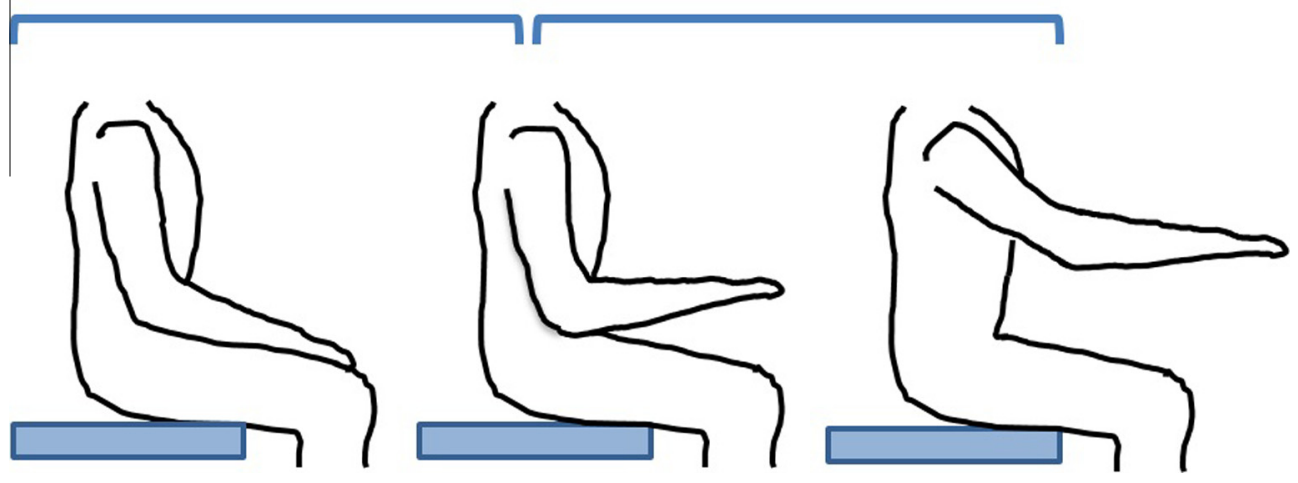

Fig. 1. Proposed division of the reaching movement based on postural control demands. 
middle cerebral artery territory, confirmed by neuroimaging; a time evolution up to 6 months and a score between 30 and 50 of the Fugl-Meyer Assessment Scale (moderately impaired) (Scheidt et al., 2007). As exclusion criteria were considered: hemispatial neglect; visual (uncorrected), perceptual or cognitive deficits; and active range of motion of the contralesional shoulder and elbow joints inferior to $15^{\circ}$ (Zackowski et al., 2004).

Post-stroke group's characterization, namely age, gender, height, weight, time post stroke and location of lesion is presented in Table 1.

\subsection{Instruments}

Surface electromyography (sEMG) was recorded using two bioPLUX $^{\circledR}$ (Plux, Portugal) devices with a sampling frequency of $1000 \mathrm{~Hz}$, common mode rejection ratio $110 \mathrm{~dB}$, input impedance greater than $100 \mathrm{M} \Omega$ and a 12 bits analog-to-digital signal conversion. The bipolar sensor configuration was selected and autoadhesive pediatric electrodes were used (Correia et al., 2004; Matias et al., 2006). The sEMG signals were analyzed through the AcqKnowledge Analysis Software version 3.9 (Biopac Systems, Inc., Goleta, USA).

Skin impedance was measured using the Noraxon ${ }^{\circledR}$ Impedance Checker system (Noraxon, Scottsdale, Arizona).

For kinematic data acquisition, four cameras (Sony Handicam DCR-HC53, Lisboa, Portugal), with a sampling frequency of $50 \mathrm{~Hz}$ were used. APAS Software (Ariel Performance Analysis System, Ariel Dynamics Inc., Canyon, USA), version 12.1.0.10, was used for data analysis. This software allows to analyze data captured from multiple cameras simultaneously, performing 3D biomechanical analysis automatically through the use of specific markers and direct linear transformation (DLT) algorithm (Abdel-Aziz et al., 1971).

\subsection{Experimental procedures}

Each subject was assessed in sitting position without trunk support. Seat height was adjusted to each participant's lower leg length, measured from the lateral line of the knee joint to the ground. At the initial position, $75 \%$ of the thigh length was seat supported (Michaelsen et al., 2001). Participants were barefoot. One height adjustable table was placed anteriorly, at the level of each subject's iliac crests. A juice glass $(5.5 \mathrm{~cm}$ of diameter) was placed on it, according to each subject's anatomical reaching distance, measured from the acromion to the thumb metacarpophalangeal joint (Reisman et al., 2006; Vandenberghe et al., 2010). Before the beginning of the task, subjects were instructed to position with the following parameters: $0^{\circ}$ of flexion/extension/ internal rotation of the shoulder; $100^{\circ}$ of flexion of the elbow; forearm in pronation; and the palm of the hand resting on thigh.

Two reaching movements were performed, one on the sagittal plane and another considering the scapular plane $\left(30^{\circ}\right.$ from the frontal plane). These planes were chosen because the functional reaching movements occur mainly in such planes. When evaluat- ing the movement performed at the sagittal plane, the glass was positioned in front of the ipsilateral shoulder, and when evaluating the scapular plane it was placed, also ipsilateraly, at $30^{\circ}$ from the frontal plane. Subjects were instructed, after a verbal cue, to reach and grasp the glass. Three valid repetitions were executed, with an interval of one minute each. All the verbal commands were given equitatively and by the same researcher.

Surface EMG was recorded from seven trunk and upper-limb muscles, namely the latissimus dorsi (LD), sternal head of pectoralis major (PM), anterior (AD), and posterior (PD) segments of deltoid, biceps brachii (BB), brachioradialis (BR), and triceps brachii lateral head (TRIlat).

Prior to data collection, the skin was shaved and wiped down with alcohol, after which skin impedance was measured and confirmed less than $5 \mathrm{k} \Omega$. Disposable pediatric $\mathrm{Ag} / \mathrm{AgCl}$ electrodes, with a skin contact surface of $10 \mathrm{~mm}^{2}$, and inter-electrode distance of $20 \mathrm{~mm}$, were placed parallel to the muscle fibers and according with SENIAM references, Table 2 (Hermens et al., 2000). Electrode placement was confirmed by voluntary muscle contraction. Ground electrodes were placed over both olecraneums (Correia et al., 2004; Hermens et al., 2000).

In the present study, kinematic analysis was only used to assess movement onset and offset, as well as to assess maximum elbow flexion. The selected acquisition setup involved the placement of the cameras at a $4 \mathrm{~m}$ distance from the subject, two in the frontal plane and two at a $45^{\circ}$ between the frontal and the sagittal planes from each shoulder (Vandenberghe et al., 2010).

The EMG and kinematic data were synchronized by a simultaneous trigger input signal (led).

\subsection{Data processing}

Kinematic data was digitally low-pass filtered using a second order Butterworth filter with a cutoff frequency of $7 \mathrm{~Hz}$, and analyzed in terms of hand peak velocity and maximum elbow flexion. Movement onset an offset were defined as the times at which third metacarpus' tangential velocity exceed or fell below $10 \%$ of its maximal value, respectively (Cirstea and Levin, 2000). Maximum elbow flexion was defined as the lowest angle between the arm and forearm segments.

The EMG data was offline processed with AcqKnowledge 3.9.0 software (Biopac Systems, Inc., California, USA). Signals were band-pass filtered at $20-500 \mathrm{~Hz}$, amplified and root-mean-square (RMS) processed for consecutive segments of $100 \mathrm{~ms}$. Muscular onset was determined as the time when the EMG activity exceeded the baseline for three standard deviations, for a minimum period of 50 ms (Hodges et al., 1996).

The reach movement was divided into two sub-phases, using the kinematic data. The first sub-phase was defined as the interval between movement onset (velocity exceeded $10 \%$ of its maximum during the task), until maximum elbow flexion, in this sub-phase muscles of shoulder girdle act to maintain postural stability, while muscles of the elbow joint act to produce movement of the distal segments. The second sub-phase corresponds to the interval

Table 1

Post-stroke group characterization regarding age, gender, weight $(\mathrm{kg})$, height $(\mathrm{m})$, time post stroke (years) and location of lesion.

\begin{tabular}{|c|c|c|c|c|c|c|c|c|c|}
\hline & \multicolumn{9}{|c|}{ Post-stroke group } \\
\hline & A & B & C & $\mathrm{D}$ & E & $\mathrm{F}$ & G & $\mathrm{H}$ & I \\
\hline Age/gender & $64 / \mathrm{M}$ & $57 / \mathrm{M}$ & $47 / F$ & $45 / \mathrm{M}$ & $45 / F$ & 49/M & $72 / \mathrm{M}$ & $53 / F$ & $63 / \mathrm{M}$ \\
\hline Weight (kg) & 82 & 105 & 70 & 75 & 60 & 72 & 68 & 69.3 & 74 \\
\hline Height $(\mathrm{m})$ & 1.70 & 1.69 & 1.63 & 1.75 & 1.63 & 1.68 & 1.65 & 1.58 & 1.65 \\
\hline Time post stroke (years) & 1 & 8 & 1 & 1 & 4 & 5 & 2 & 2 & 8 \\
\hline Location of lesion & LMCA & RMCA & RMCA & LMCA & LMCA & RMCA & LMCA & RMCA & RMCA \\
\hline
\end{tabular}

A-I represent each subject; M - male; F - female; LMCA - left medial cerebral artery; RMCA - right medial cerebral artery. 
Table 2

Anatomical references used to locate the electrodes. Electrode locations were confirmed by palpation of the muscular belly with the subject in the test position.

\begin{tabular}{ll}
\hline Muscle & Electrode placement \\
\hline LD & $\begin{array}{l}\text { One centimetre laterally to the lateral edge of the scapula } \\
\text { Two fingers below the collarbone and at two fingers from the } \\
\text { sternum } \\
\text { One finger width distal and anterior to the acromion, in the } \\
\text { direction of the line between the acromion and the thumb } \\
\text { Two finger width behind the angle of the acromion, in the } \\
\text { direction of the line between the acromion and the little finger }\end{array}$ \\
PD & $\begin{array}{l}\text { On the line between the medial acromion and the fossa cubit } \\
\text { at } 1 / 3 \text { from the fossa cubit }\end{array}$ \\
BB & $\begin{array}{l}\text { Most prominent bulge of the muscle } \\
\text { At } 50 \% \text { on the line between the posterior crista of the acromion } \\
\text { and the olecranon at } 2 \text { finger widths lateral to the line } \\
\text { BR }\end{array}$ \\
BRIlat & Both olecraneums \\
Ground &
\end{tabular}

between maximum elbow flexion until movement offset (velocity fell below $10 \%$ of its maximum during the task), where shoulder girdle and elbow muscles act to produce upper limb forward displacement. In each sub-phase, the antagonist co-activation ratios of the muscle pairs (LD/PM, PD/AD, TRIlat/BB, TRIlat/BR) were evaluated. The antagonist co-activation ratio was calculated according to (Kellis et al., 2003):

$C(\%)=\frac{\text { antagonist activity }}{\text { agonist }+ \text { antagonist activity }} \times 100$

This approach provides an estimate of the relative activation of the pair of muscles, as well as the magnitude of the co-activation.

Considering that differences in upper limb motor patterns have been demonstrated between dominant and non-dominant limbs, and that post-stroke subjects tend to use the ipsilesional limb as dominant limb, the values obtained from the ipsilesional limb were compared to those obtained from the dominant limb while the values obtained from the contralesional limb were compared to those obtained from non-dominant limb (Sainburg et al., 2000; Sainburg et al., 2004; Sainburg, 2002).

\subsection{Statistics}

Statistical Analysis was performed using IBM SPSS Statistics software v20 with a significance level of 0.05 . Due to the small number of participants ( $n=9$ per group), Mann-Whitney U test was used to compare healthy versus post-stroke groups, and Friedman ANOVA test, with Dunn's test as Post-Hoc, to compare sides and planes within each group. Median and interquartile deviation were used as descriptive statistics (Marôco, 2010).

\section{Results}

The following results characterize the antagonist co-activation ratios of the muscle pairs LD/PM; $\mathrm{AD} / \mathrm{PD}$; TRlat/BB and TRlat/BR of the ipsilesional limb of post-stroke subjects compared to the dominant limb of the healthy group and the contralesional limb compared to the non-dominant limb. Still, no statistical differences were observed between dominant and non-dominant limbs of healthy subjects (data not shown).

3.1. Antagonist co-activation ratios in the ipsilesional limb of poststroke subjects in the first sub-phase of the reaching movement

In the first sub-phase of the reaching movement, the antagonist co-activation ratio of the muscle pair LD/PM was significantly lower in the post-stroke's ipsilesional limb, in relation to the dominant limb of healthy subjects, when the movement was performed on the sagittal plane $(p=0.04)$. Conversely, on the scapular plane of movement no statistical differences $(p>0.05)$ were observed. The antagonist co-activation ratios of the other muscle pairs (PD/AD, TRIlat/BB and TRIlat/BR) did not evidenced a statistical different $(p>0.05)$ behavior between both groups (Table 3$)$.

\subsection{Antagonist co-activation ratios in the contralesional limb of post-stroke subjects in the first sub-phase of the reaching movement}

In the first sub-phase of the reaching movement, the contralesional limb showed a significant increase of the antagonist coactivation ratio of the muscle pair TRllat/BB when the movement was performed on the sagittal plane $(p=0.02)$. Conversely, no significant differences $(p>0.05)$ of the antagonist co-activation of the muscle pairs LD/PM, PD/AD and TRIat/BR were detected between the contralesional limb of post-stroke subjects and the nondominant limb of healthy subjects for both sagittal and scapular planes (Table 3).

\subsection{Antagonist co-activation ratios in the ipsilesional limb of post-stroke subjects in the second sub-phase of the reaching movement}

In the second sub-phase of the reaching movement, no significant differences $(p>0.05)$ were observed between the ipsilesional limb of post-stroke subjects and the dominant limb of the healthy group in all of the studied muscle pairs, namely LD/PM, PD/AD, TRIat/BB and TRIat/BR (Table 4)

3.4. Antagonist co-activation ratios in the contralesional limb of poststroke subjects in the second sub-phase of the reach movement

In the second sub-phase of the reaching movement, the contralesional limb of post-stroke subjects presented higher antagonist co-activation ratios of the muscle pairs LD/PM $(p=0.019)$, in the sagittal plane of movement, and of the $\mathrm{PD} / \mathrm{AD}$ pair in both sagittal $(p=0.008)$ and scapular $(p=0.04)$ planes of movement, comparing to non-dominant limb of healthy subjects. The other analyzed muscle pairs, TRIlat/BB and TRIlat/BR showed no statistical differences $(p>0.05)$ (Table 4$)$.

\section{Discussion}

\subsection{Antagonist co-activation ratio in the ipsilesional limb of post-stroke subjects}

In this study we demonstrated that the ipsilesional limb of poststroke subjects shows an altered antagonist co-activation ratio of the LD/PM muscle pair in the first sub-phase of the reaching movement, when performed in the sagittal plane. Moreover, no significant differences were observed between the ipsilesional limb of post-stroke subjects and the dominant limb of healthy subjects in the second sub-phase of the movement, in both sagittal and scapular planes. These findings are in agreement with our postulated hypothesis, i.e., that the ipsilesional limb of post-stroke subjects presents a motor dysfunction of postural muscles. Given that the first sub-phase of the reaching task imposes a higher demand of the proximal postural control, in relation to the second subphase that imposes higher demands in terms of proximal mobility, the different behavior between the ipsilesional side of stroke subjects and healthy subjects may be explained by the dysfunctional neuronal systems that present mostly an ispsilateral disposal/ projection. Moreover, the fact that stroke subjects evidenced subcortical lesions compatible with alterations of the cortical-reticular circuits reinforces this possibility. Concerning the upper-limb analysis, little or no evidence corroborant with these findings 
Table 3

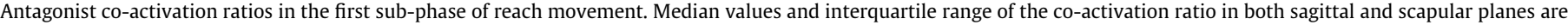

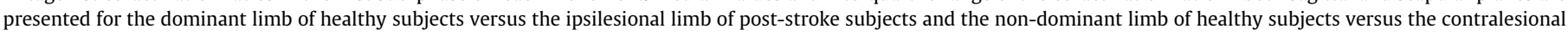
limb of post-stroke subjects, for LD/PM, PD/AD, TRlat/BB and TRlat/BR muscle pairs.

\begin{tabular}{|c|c|c|c|c|c|c|}
\hline & \multicolumn{3}{|c|}{ Median (interquartil range) } & \multicolumn{3}{|l|}{ Median (Interquartil Range) } \\
\hline & Healthy dominant limb & Ipsilesional limb & $p$-value & Healthy non-dominant limb & Contralesional limb & $p$-value \\
\hline \multicolumn{7}{|c|}{ Muscle pairs } \\
\hline $\mathrm{LD} / \mathrm{PM}$ & $57.7( \pm 11.4)$ & $46.0( \pm 8.6)$ & $0.04^{*}$ & $46.1( \pm 9.7)$ & $54.1( \pm 8.2)$ & 0.094 \\
\hline $\mathrm{PD} / \mathrm{AD}$ & $21.0( \pm 9.7)$ & $23.7( \pm 7.5)$ & 0.546 & $20.7( \pm 11.7)$ & $39.8( \pm 16.2)$ & 0.190 \\
\hline TRIat/BB & $41.8( \pm 12.0)$ & $42.4( \pm 14.3)$ & 0.489 & $30.6( \pm 6.48)$ & $46.4( \pm 11.2)$ & $0.02^{*}$ \\
\hline TRIlat/BR & $35.0( \pm 7.6)$ & $26.5( \pm 14.1)$ & 0.796 & $34.9( \pm 21.9)$ & $54.0( \pm 20.1)$ & 0.340 \\
\hline \multicolumn{7}{|c|}{ Scapular plane } \\
\hline LD/PM & $56.7( \pm 8.0)$ & $56.7( \pm 11.6)$ & 1.000 & $54.3( \pm 10.3)$ & $59.0( \pm 4.6)$ & 0.136 \\
\hline $\mathrm{PD} / \mathrm{AD}$ & $41.0( \pm 11.9)$ & $36.3( \pm 18.9)$ & 0.937 & $31.8( \pm 21.4)$ & $35.1( \pm 18.5)$ & 0.0605 \\
\hline TRIlat/BB & $47.0( \pm 12.3)$ & $42.3( \pm 12.3)$ & 0.863 & $40.8( \pm 8.3)$ & $46.0( \pm 4.4)$ & 0.258 \\
\hline TRIlat/BR & $32.9( \pm 10.7)$ & $27.9( \pm 13.0)$ & 0.546 & $34.7( \pm 18.8)$ & $55.5( \pm 20.4)$ & 0.605 \\
\hline
\end{tabular}

Table 4

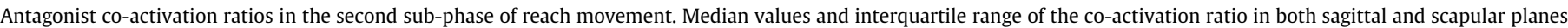

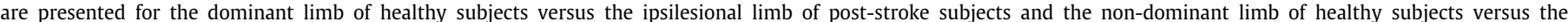
contralesional limb of post-stroke subjects, for LD/PM, PD/AD, TRlat/BB and TRlat/BR muscle pairs.

\begin{tabular}{|c|c|c|c|c|c|c|}
\hline & \multicolumn{3}{|c|}{$\underline{\text { Median (interquartil range) }}$} & \multicolumn{3}{|l|}{ Median (Interquartil Range) } \\
\hline & Healthy dominant limb & Ipsilesional limb & $p$-value & Healthy non-dominant limb & Contralesional limb & $p$-value \\
\hline \multicolumn{7}{|c|}{ Muscle pairs } \\
\hline LD/PM & $45.5( \pm 14.4)$ & $45.3( \pm 10.8)$ & 0.29 & $42.6( \pm 9.0)$ & $56.6( \pm 10.7)$ & 0.019 \\
\hline $\mathrm{PD} / \mathrm{AD}$ & $7.2( \pm 3.6)$ & $11.0( \pm 4.0)$ & 0.16 & $8.2( \pm 2.0)$ & $30.6( \pm 8.8)$ & 0.008 \\
\hline TRIlat/BB & $51.9( \pm 10.2)$ & $46.2( \pm 14.9)$ & 0.61 & $59.9( \pm 7.6)$ & $53.2( \pm 14.8)$ & 0.297 \\
\hline TRIlat/BR & $45.0( \pm 7.7)$ & $58.9( \pm 14.8)$ & 0.26 & $58.2( \pm 13.0)$ & $46.2( \pm 17.2)$ & 0.489 \\
\hline \multicolumn{7}{|c|}{ Scapular plane } \\
\hline $\mathrm{LD} / \mathrm{PM}$ & $55.2( \pm 9.0)$ & $53.9( \pm 9.7)$ & 0.44 & $53.6( \pm 6.1)$ & $58.9( \pm 8.1)$ & 0.094 \\
\hline $\mathrm{PD} / \mathrm{AD}$ & $12.6( \pm 4.5)$ & $14.7( \pm 4.2)$ & 0.93 & $13.9( \pm 5.7)$ & $26.6( \pm 7.5)$ & 0.04 \\
\hline TRIlat/BB & $57.3( \pm 15.9)$ & $43.6( \pm 17.6)$ & 0.29 & $59.7( \pm 7.2)$ & $48.5( \pm 10.6)$ & 0.190 \\
\hline TRIlat/BR & $55.2( \pm 14.5)$ & $60.6( \pm 11.5)$ & 0.73 & $60.3( \pm 13.4)$ & $50.6( \pm 16.9)$ & 0.489 \\
\hline
\end{tabular}

was found. On the other hand, regarding the lower-limb, atypical antagonist co-activation ratios in the ipsilesional limb were already reported, but causes were attributed to a compensatory mechanism, rather than resulting from the lesion itself (Lamontagne et al., 2000). Recently, an altered antagonist coactivation ratio was found in the ipsilesional lower-limbs of stroke subjects, an effect that was attributed to a possible dysfunction of ipsilaterally-descending pathways (Silva et al., 2012a,b; Sousa et al., 2013)

The fact that, when the reaching movement was performed in the scapular plane, the antagonist co-activation ratio of the ispilesional limb evidenced a similar behavior when compared to the healthy subjects, might be explained by the influence of scapula's alignment in the mechanical efficiency of proximal postural control for reaching. As stated by Kibler (1998), in this particular alignment there is an enhanced biomechanical relation between the scapula and the thoracic wall, which contributes to improve postural control function and, thus, imposes less challenge to LD/PM muscle pair. This perspective about the supremacy of the scapular plane of movement for stability muscular recruitment is also hypothesized by Matias et al. (Kibler, 1998; Matias et al., 2006).

Considering that: (1) the second sub-phase of the reaching movement is more mobility-demanding rather than stability-demanding; (2) the fact that the differences between the ipsilesional limb stroke subjects and the dominant limb of healthy subjects in the behavior of the LD/PM muscle pair were no longer observed in the second subphase of the movement; and (3) that the muscle pairs whose major role is mobility did not show significant differences between the ipsilesional limb of stroke subjects when compared to the dominant limb of healthy subjects; strengthen the idea that the ipsilesional limb presents postural control deficits.

\subsection{Antagonist co-activation ratio in the contralesional limb of post-stroke subjects}

Concerning the analysis of antagonist co-activation ratios at the contralesional side, our results are in agreement with the widespread scientific evidence that stroke subjects exhibit atypical co-activation levels between muscle pairs, when compared to healthy subjects (Higginson et al., 2006; Lamontagne et al., 2002, 2000). However, it is important to explore this overall tendency to higher co-activation levels, relating the role of the muscle pairs according to the movement sub-phase. So, at the first sub-phase of the movement, statistical differences between groups were only observed in the muscle pair TRIlat/BB, which is considered to be the mobility pair for the elbow joint. Indeed, at this sub-phase, the elbow flexion demands the coactivation of these muscles (flexors/extensors), which is, in these participants, altered. This finding may be explained by the lesion at the corticospinal system, which influences the activity of agonist muscles. Since impairment of this system is associated to lower muscle activation capacity during movement tasks, the increased antagonist co-activation ratio can be sustained by this influence.

When analyzing the second sub-phase, and according with the above stated, it is important to understand that, since at this sub-phase, the shoulder is the joint which contributes the most to provide the upper-limb displacement towards the goal, the muscles at this joint need to be considered mainly mobility orientated. So, statistical differences were only observed in the muscle pairs $\mathrm{LD} / \mathrm{PM}$ (at the saggital plane) and $\mathrm{PD} / \mathrm{AD}$ (at both planes). It is important to highlight that although proximal muscles frequently assume a stability role, the reaching gesture incorporates continuous shifts in their roles, according to the upper-limb's segment presenting higher mobility. 


\subsection{Limitations}

Despite, like other authors (Lamontagne et al., 2000), we opted to use absolute EMG levels, this methodological decision has as drawback the fact that, as we did not measure the thickness of the subcutaneous fat layer, we cannot guarantee that this variable did not influenced the co-activation levels obtained. Nevertheless the EMG normalization in post-stroke subjects can be questionable given the abnormal EMG pattern frequently exhibited by these subjects. As a consequence, the EMG normalization in post-stroke subjects can lead to distortion of co-activation values and to higher variability (Yang et al., 1984)

\subsection{Clinical implications}

These results should be taken into account in the rehabilitation field, i.e., clinicians should be aware to the fact that the ipsilesional side of stroke subjects may also present a motor control dysfunction. For this reason ipsilesional side of stroke subjects should not be overlooked and involved in the rehabilitation strategy.

On the other hand, the possible adverse influence of the atypical behavior found in the ipsilesional side (regarding postural control function) over the contralesional side has not yet been fully explored. The pertinence of such (to be addressed in future research) is based on the knowledge that the type II fibers present bilateral connections, justifying thus, the possible interdependence between both sides of the stroke subjects (Jankowska et al., 2005).

\section{Conclusion}

Our findings demonstrate that, in post-stroke subjects, the ipsilesional upper limb might show signs of postural control dysfunction, whereas the contralesional upper limb might have signs of movement dysfunction.

Although further research is still needed, the global motor dysfunction after an unilateral brain lesion has been gaining evidence.

\section{Acknowledgements}

The first author would like to thank to Escola Superior de Tecnologias da Saúde do Porto - Instituto Politécnico do Porto, for the support through the conceded grant. The authors would like to thank the Foundation for Science and Technology of Portugal for their support of some of the PhD students involved in this article (SFRH/BD/ $61396 / 2009$ and SFRH/BD/60929/2009). Additionally, the authors would like to acknowledge the contribution of all volunteers that took part of the testing procedures. A special thanks to all patients for their efforts and continuous support of this research. 


\section{References}

Abdel-Aziz YI, Karara HM. Direct linear transformation from comparator coordinates into object space coordinates in close-range photogrammetry. In: Paper presented at the symposium on close-range photogrammetry. Falls Church, VA; 1971.

Bobath, B. Adult hemiplegia. Evaluation and treatment. 3rd ed. Heinemann Medical, Oxford; 1990.

Bourbonnais D, Vanden Noven S. Weakness in patients with hemiparesis. Am J Occup Ther 1989;43:313-9.

Burke D. Spasticity as an adaptation to pyramidal tract injury. Adv Neuro 1988;47:401-22.

Champiom J, Barber C, Lynch-Ellerington M. Recovery of upper limb function. In: Sue Raine, L.M., Lynch-Ellerington Mary (Eds.), Bobath concept. theory and clinical practice in neurological rehabilitation. Wiley-Blackwell; 2009.

Cirstea MC, Levin MF. Compensatory strategies for reaching in stroke. Brain 2000;123(5):940-53.

Correia PP, Mil-Homens P. A Electromiografia no Estudo do Movimento Humano. Lisboa; 2004

Desrosiers Johanne, Bourbonnais Daniel, Bravo Gina, Roy Pierre-Michel, Guay Manon. Performance of the 'unaffected' upper extremity of elderly stroke patients. Stroke 1996;27(9):1564-70. http://dx.doi.org/10.1161/ 01.str.27.9.1564.

Dickstein R, Shefi S, Marcovitz E, Villa Y. Anticipatory postural adjustment in selected trunk muscles in poststroke hemiparetic patients. Arch Phys Med Rehabil 2004;85:261-7.

el-Abd MA, Ibrahim IK, Dietz V. Impaired activation pattern in antagonistic elbow muscles of patients with spastic hemiparesis: contribution to movement disorder. Electromyogr Clin Neurophysiol 1993;33(4):247-55.

Fellows SJ, Kaus C, Ross HF, Thilmann AF. Agonist and antagonist EMG activation during isometric torque development at the elbow in spastic hemiparesis. Electroencephalogr Clin Neurophysiol/Evoked Potentials Section 1994;93(2):106-12. doi:10.1016/0168-5597(94)90073-6.

Geuze Reint H. Postural control in children with developmental coordination disorder. Neural Plasticity 2005;12(2-3):183-96. http://dx.doi.org/10.1155 np.2005.183.

Gowland Carolyn, deBruin Hubert, Basmajian John V, Plews Nancy, Burcea Ion. Agonist and antagonist activity during voluntary upper-limb movement in patients with stroke. Phys Ther 1992;72(9):624-33.

Hammond MC, Fitts SS, Kraft GH, Nutter PB, Trotter MJ, Robinson LM. Cocontraction in the hemiparetic forearm: quantitative EMG evaluation. Arch Phys Med Rehabil 1988;69(5):348-51.

Hermens Hermie J, Freriks Bart, Disselhorst-Klug Catherine, Rau Günter. Development of recommendations for SEMG sensors and sensor placement procedures. J Electromyogr Kinesiol 2000;10(5):361-74. doi:10.1016/S10506411(00)00027-4.

Higginson JS, Zajac FE, Neptune RR, Kautz SA, Delp SL. Muscle contributions to support during gait in an individual with post-stroke hemiparesis. J Biomech 2006:39(10):1769-77. doi:10.1016/j.jbiomech.2005.05.032.

Hodges Paul W, Bui Bang H. A comparison of computer-based methods for the determination of onset of muscle contraction using electromyography. Electroencephalogr Clin Neurophysiol/Electromyogr Motor Control 1996:101(6):511-9. doi:10.1016/S0921-884X(96)95190-5.

Hughes AM, Freeman CT, Burridge JH, Chappell PH, Lewin PL, Pickering RM, Rogers E. Shoulder and elbow muscle activity during fully supported trajectory tracking in neurologically intact older people. J Electromyogr Kinesiol 1996:19(6):1025-34. doi:10.1016/j.jelekin.2008.09.015.

Hughes AM, Freeman CT, Burridge JH, Chappell PH, Lewin PL, Rogers E. Shoulder and elbow muscle activity during fully supported trajectory tracking in people who have had a stroke. J Electromyogr Kinesiol 2010:20(3):465-76. doi:10.1016 j.jelekin.2009.08.001.

Jankowska E, Edgley SA, Krutki P, Hammar I. Functional differentiation and organization of feline midlumbar commissural interneurones. J Physiol 2005;565(2):645-58. http://dx.doi.org/10.1113/jphysiol.2005.083014.

Kellis E, Arabatzi F, Papadopoulos C. Muscle co-activation around the knee in drop jumping using the co-contraction index. J Electromyogr Kinesiol 2003;13(3):229-38.

Kibler WBen. The role of the scapula in athletic shoulder function. Am J Sports Med 1998;26(2):325-37.

Lamontagne Anouk, Richards Carol L, Malouin Francine. Coactivation during gait as an adaptive behavior after stroke. J Electromyogr Kinesiol 2000:10(6):407-15. doi:10.1016/S1050-6411(00)00028-6.

Lamontagne A, Malouin F, Richards CL, Dumas F. Mechanisms of disturbed motor control in ankle weakness during gait after stroke. Gait Posture 2002;15(3):244-55. doi:10.1016/S0966-6362(01)00190-4.

Marôco J. Análise estatística com o PASW. Statistics, 1 ed. ReportNumber; 2010.

Matias R, Batata D, Morais D, Miguel J, Estiveira R. Estudo do Comportamento Motor dos Músculos Deltóide, Trapézio, e Grande Dentado Durante a Elevação do Braço em Sujeitos Asssintomáticos. EssFisioOnline 2006;2(4):3-23.

Matsuyama Kiyoji, Mori Futoshi, Nakajima Katsumi, Drew Trevor, Aoki Mamoru, Mori Shigemi. Locomotor role of the corticoreticular-reticulospinal-spinal interneuronal system. In: Shigemi Mori, DGS, Mario W. (Eds.), Progress in Brain Research. Elsevier; vol. 143, 2004. p. 239-49.
Michaelsen Stella M, Luta Anamaria, Roby-Brami Agnès, Levin Mindy F. Effect of trunk restraint on the recovery of reaching movements in hemiparetic patients. Stroke 2001;32(8):1875-83. http://dx.doi.org/10.1161/01.str.32.8.1875.

Patel S, Hughes R, Hester T, Stein J, Akay M, Dy J, Bonato P. Tracking motor recovery in stroke survivors undergoing rehabilitation using wearable technology. In: Paper presented at the engineering in medicine and biology society (EMBC), 2010 annual international conference of the IEEEl; 2010, August $312010-$ September 42010.

Reisman Darcy S, Scholz John P. Workspace location influences joint coordination during reaching in post-stroke hemiparesis. Exp Brain Res 2006;170(2):265-76. http://dx.doi.org/10.1007/s00221-005-0209-5.

Sainburg Robert. Evidence for a dynamic-dominance hypothesis of handedness. Exp Brain Res 2002;142(2):241-58. http://dx.doi.org/10.1007/s00221-001-0913-8.

Sainburg RL, Kalakanis D. Differences in control of limb dynamics during dominant and nondominant arm reaching. J Neurophysiol 2000;83(5):2661-75.

Sainburg RL, Schaefer S. Interlimb differences in control of movement extent. J Neurophysiol 2004;92:1374-83.

Scheidt Robert A, Stoeckmann Tina. Reach adaptation and final position control amid environmental uncertainty after stroke. J Neurophysiol 2007;97(4):2824-36. http://dx.doi.org/10.1152/jn.00870.2006.

Schepens Bénédicte, Drew Trevor. Independent and convergent signals from the pontomedullary reticular formation contribute to the control of posture and movement during reaching in the cat. J Neurophysiol 2004;92(4):2217-38 http://dx.doi.org/10.1152/in.01189.2003.

Schepens Bénédicte, Stapley Paul, Drew Trevor. Neurons in the pontomedullary reticular formation signal posture and movement both as an integrated behavior and independently. J Neurophysiol 2008;100(4):2235-53. http:/ dx.doi.org/10.1152/in.01381.2007.

Shumway-Cook A, Woollacott M. Normal reach grasp and manipulation motor control - translating research into clinical practice. Lippincott Williams \& Wilkins; 3rd ed.; 2007. p. 443-67.

Silva A, Sousa AS, Pinheiro R, Tavares JM, Santos R, Sousa F. Soleus activity in poststroke subjects: movement sequence from standing to sitting. Somatosens Mot Res 2012a;29(3):71-6. http://dx.doi.org/10.3109/08990220.2012.686935.

Silva A, Sousa AS, Tavares JM, Tinoco A, Santos R, Sousa F. Ankle dynamic in stroke patients: agonist vs. antagonist muscle relations. Somatosens Mot Res 2012b;29(4):111-6. http://dx.doi.org/10.3109/08990220.2012.715099.

Sousa, Andreia SP, Silva Augusta, Santos Rubim, Sousa Filipa, Tavares João Manuel RS. Interlimb coordination during the stance phase of gaitin subjects with stroke. Arch Phys Med Rehab; 2013. doi:10.1016/j.apmr.2013.06.032.

Stoeckmann Tina M, Sullivan Katherine J, Scheidt Robert A. Elastic, viscous, and mass load effects on poststroke muscle recruitment and co-contraction during reaching: a pilot study. Phys Ther 2009;89(7):665-78. http://dx.doi.org/ $10.2522 /$ ptj.20080128.

Sunderland Alan, Bowers Mark P, Sluman Stella-Marie, Wilcock David J, Ardron Mark E. Impaired dexterity of the ipsilateral hand after stroke and the relationship to cognitive deficit. Stroke 1999;30(5):949-55. http://dx.doi.org/ 10.1161/01.str.30.5.949.

Tate D. The state of rehabilitation research: art or science? Arch Phys Med Rehab 2006;87:160-6.

Vandenberghe Annelies, Levin Oron, De Schutter Joris, Swinnen Stephan, Jonkers Ilse. Three-dimensional reaching tasks: Effect of reaching height and width on upper limb kinematics and muscle activity. Gait Posture 2010;32(4):500-7. doi:10.1016/j.gaitpost.2010.07.009.

WHO. Stroke, cerebrovascular accident; 2011.

Yang JF, Winter DA. Electromyographic amplitude normalization methods: improving their sensitivity as diagnostic tools in gait analysis. Arch Phys Ther Med Rehab 1984;65:517-21.

Yanga N, Zhanga M, Huangb C, Dewen J. Synergic analysis of upper limb targetreaching movements. J Biomech 2002;35:739-46.

Zackowski KM, Dromerick AW, Sahrmann SA, Thach WT, Bastian AJ. How do strength, sensation, spasticity and joint individuation relate to the reaching deficits of people with chronic hemiparesis? Brain 2004;127(5):1035-46. http://dx.doi.org/10.1093/brain/awh116.

Zattara M, Bouisset S. Posturo-kinetic organisation during the early phase of voluntary upper limb movement. 1 Normal subjects. J Neurol Neurosurg Psychiatry 1988;51:956-65. 


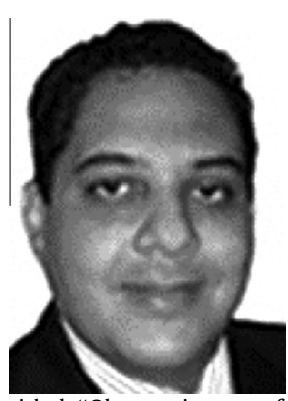

Antonio J. Salazar is a researcher of the Institute for Systems and Computer Engineering of Porto, currently pursuing doctoral studies with support from the Foundation for Science and Technology of Portugal, at the University of Porto, Portugal were he focuses on design for testability and built-in testing instruments for wearable monitoring solutions. He graduated with a B.Sc. in Electrical Engineering from the California Institute of Technology were he had the opportunity to work on the Neuro-probe project within the Pine Laboratory. Obtained his M.Sc. in Electrical and Computer Engineering from the University of California, Santa Barbara, with a thesis titled "Observations on forced variable re-introduction for BDD simplification". Holds the position of Associate Professor at the University Simon Bolivar were he co-founded the Center for Assistive Technology dedicated to the development of low-cost solutions for individuals with motor and/or linguistic compromise. Author of several international conferences and peer reviewed papers.

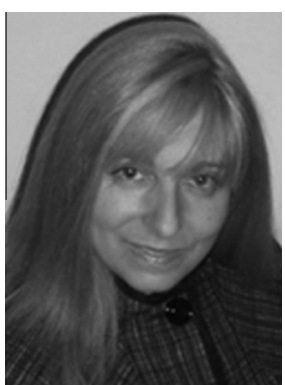

Carla M. Borges is currently pursuing doctoral studies at the University of Porto, Portugal in Biomedical Engineering were she focuses on movement patterns of the upper limb in human subjects using accelerometry and electromyography. She graduated as an Electronic Engineering from the University Simón Bolívar with a thesis titled "Implementation of load sensitive preamplifier for Alpha spectrometry", where she works with the group of Nuclear Physics. Obtained her Master Degree in the same specialty from the University Simón Bolivar, Venezuela with a thesis titled "Methodology proposal for processing and acquisition of accelerometry for Stroke rehabilitation system". Had the position of Instructor at the University Simón Bolívar was involved in research on Biomechanical models and Hospital management.

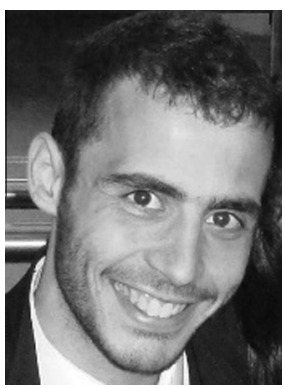

Carlos Crasto received in 2013 the MSc degree in Physiotherapy - Manual Orthopedic Therapy from School of Applied Health Sciences, Portugal, where he is currently an Assistant Professor in this field. He also worked at the statistical department of the same institution.

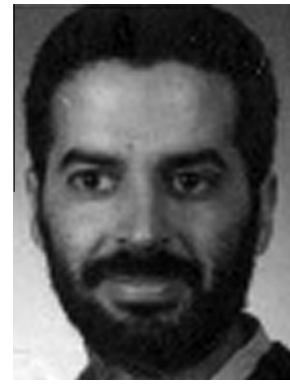

Miguel V. Correia received the MSc. and $\mathrm{PhD}$ degrees in electrical and computer engineering from the Faculty of Engineering of the University of Porto (FEUP), Portugal, in 1995 and 2001, respectively, in the fields of Industrial Automation and Computer Vision. Currently, he is a full-professor at the Department of Electrical and Computer Engineering of FEUP and also a member of the research team of the Optoelectronics and Electronic Systems Unit of INESC-TEC Porto. Co-funder and technical advisor of Kinematix., a company specialized in the design and manufacture of innovative electronic devices for the health, sports and industrial markets.

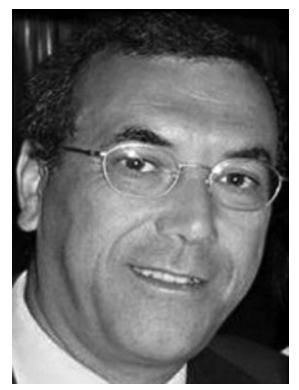

J. Paulo Vilas-Boas full professor at the Faculty of Sport and Porto Biomechanics laboratory of the University of Porto. Doctor in Sport Sciences (Ph.D), specialty in Sports Biomechanics, at the University of Porto, 1993. Member of the Steering Group "Swimming" of the World Commission for Science in Sports (WCSS-ICSSPE - UNESCO) and of the Board of the Portuguese Society of Biomechanics. Author of several international conferences and peer reviewed papers; he was recently the chairman of the XXIXth International Symposium of the International Society of Biomechanics in Sports.

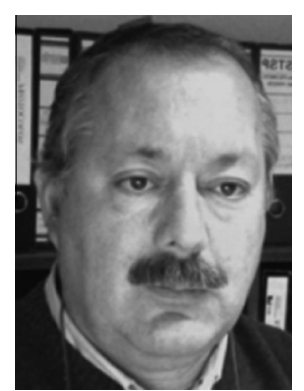

Rubim S. Santos received the B.Sc. and MSc. degrees in Physics from the Faculty of Sciences of the University of Porto, Portugal, in 1989 and 1999, respectively. Obtained his Ph.D. in Sports Science in the field of Biomechanics, from the Faculty of Sport of the University of Porto. He is now a full professor at Escola Superior de Tecnologias da Saúde do Instituto Politécnico do Porto (ESTSP-IPP) - Portugal. Director of the Centro de Estudos e Actividade Humana (CEMAH) of ESTSP-IPP, and coordinator of the project "Reorganization of Postural Control as a Result of Afferent Modifications". Member of the scientific commission of the I International Congress of Health, held in Gaia in 2010, and the ISBS2011 - XXIX Annual International Symposium of the ISBS, held in Porto in 2011. 